\title{
Ambiances
}

anbiances Environnement sensible, architecture et espace urbain Comptes-rendus | 2014

\section{Human Space ou la recherche de l'espace perdu}

A propos de la traduction anglaise de Mensch und Raum, Otto Friedrich Bollnow, 1963

\section{Céline Bonicco-Donato}

\section{(2) OpenEdition}

\section{Journals}

Édition électronique

URL : http://journals.openedition.org/ambiances/421

DOI : 10.4000/ambiances.421

ISSN : 2266-839X

\section{Éditeur :}

Direction Générale des Patrimoines - DAPA - MCC, UMR 1563 - Ambiances Architectures Urbanités (AAU)

Référence électronique

Céline Bonicco-Donato, "Human Space ou la recherche de l'espace perdu », Ambiances [En ligne], Comptes-rendus, mis en ligne le 19 avril 2014, consulté le 22 septembre 2020. URL : http:// journals.openedition.org/ambiances/421 ; DOI : https://doi.org/10.4000/ambiances.421

Ce document a été généré automatiquement le 22 septembre 2020.

\section{c)}

Ambiances is licensed under a Creative Commons Attribution-NonCommercial-NoDerivatives 4.0 International License. 


\title{
Human Space ou la recherche de l'espace perdu
}

\author{
A propos de la traduction anglaise de Mensch und Raum, Otto Friedrich \\ Bollnow, 1963
}

\section{Céline Bonicco-Donato}

\section{RÉFÉRENCE}

Bollnow, Otto Friedrich. 2011 [1963]. Human Space. London: Hyphen Press.

1 La récente traduction anglaise de Christine Shuttleworth met enfin à la disposition du public non germanophone le magistral ouvrage de Otto Friedrich Bollnow, à l'origine $\mathrm{du}$ «tournant spatial» pris par les sciences humaines dans la seconde moitié du $\mathrm{XX}^{\mathrm{e}}$ siècle. Avec une érudition impressionnante qui n'exclut nullement la sensibilité aux petits riens de l'expérience ordinaire, convoquant aussi bien des philosophes que des psychologues en passant par les grands noms de la poésie allemande, Mensch und Raum, publié initialement en 1963, se présente comme une exploration de "la dimension spatiale de l'existence humaine » (Bollnow, 2011, p. 15). S'inscrivant dans la perspective phénoménologique, Bollnow ambitionne de consacrer à l'espace la même attention que celle accordée au temps dans la philosophie du $\mathrm{XX}^{\mathrm{e}}$ siècle, en mettant au jour ses dimensions concrètes, éprouvées et vécues, trop souvent occultées par une compréhension mathématique réductrice. Si Bollnow reconnaît que cette perspective a été notamment amorcée par Martin Heidegger, Ludwig Binswanger, Erwin Strauss, ou encore Gaston Bachelard, il se propose de la développer pour la première fois de manière systématique et cohérente. Accomplissant le même renversement que celui opéré par Henri Bergson (2009 \& 2007) qui invite son lecteur, dans l'Essai sur les données immédiates de la conscience et La pensée et le mouvant, à retrouver la durée sous le temps des horloges, Mensch und Raum entreprend de révéler l'espace vécu sous celui mesurable et quantifiable, qui n'est qu'une compréhension dérivée dont il est possible de rendre compte de la construction progressive au cours de l'histoire, notamment à 
l'âge baroque avec son exaltation de l'infini. Pour cela, Bollnow fait preuve de la même confiance envers la langue que Heidegger. Dans un éblouissant premier chapitre intitulé "La structure élémentaire de l'espace», il s'appuie non seulement sur les analyses d'Aristote dans sa Physique, mais aussi sur l'étymologie du terme allemand Raum à partir du célèbre Dictionnaire de Jacob et Wilhelm Grimm, pour montrer que l'espace était originellement compris comme un lieu clos, délimité, tracé par la main humaine dans un environnement sauvage pour lui permettre de s'y établir. Ainsi s'avère-t-il inséparable du développement de la vie humaine.

2 A cet égard, le titre anglais, Human Space, peut prêter à confusion : l'expression d'espace vécu (gelebter Raum) employée par Bollnow ne doit en aucune manière être entendue en un sens subjectif comme la manière dont l'homme éprouverait un espace déjà donné, existant indépendamment de lui. Pour le dire autrement, il n'y a pas d'un côté l'espace et de l'autre sa représentation humaine, mais de manière plus fondamentale l'espace en tant que l'homme vit en son sein et par son moyen. L'espace vécu est « l'espace concret, réel, dans lequel notre existence se déroule" (Bollnow, 2011, p. 20), de la même manière que dans Etre et temps de Heidegger, le monde ne peut s'appréhender qu'à partir du Dasein, dans la mesure où ce dernier existe fondamentalement comme êtreau-monde (Heidegger, 1986, p. 52sqq). Loin d'être un contenant neutre, homogène et isotrope, l'espace apparait ainsi fondamentalement comme une "forme d'expression, de mise à l'épreuve, et de réalisation du sujet qui vit en lui, en fait l'expérience et se comporte par rapport à lui » (Dürckeim, 1932, p. 389, repris par Bollnow, 2011, p. 21). Qu'il soit impossible d'envisager l'un sans l'autre permet de comprendre la structure du livre: dans les chapitres 2, 3 et 4, Bollnow explore avec une précision d'entomologiste la manière dont l'homme vit l'espace, avant de s'interroger dans un ultime développement sur la spatialité de l'existence humaine. En effet, si l'homme n'existe qu'en rapport avec l'espace, il est non seulement possible d'interroger son expérience de l'espace, mais aussi, de manière plus profonde, de mettre au jour la structure de son existence rendant compte de ce rapport consubstantiel, ce que Heidegger appelle un « existential » ou une modalité d'être fondamentale dans Etre et temps (Heidegger, 1986, p. 44). Ainsi, s'interroger sur l'espace revient in fine à s'interroger sur l'homme. A ce titre, Mensch und Raum se présente comme une remarquable enquête philosophique sur la singularité de l'existence humaine: son exploration des différentes strates composant l'espace vécu ne fournit pas seulement une carte de notre expérience spatiale, de ses reliefs et de ses paysages, mais dessine aussi une topographie de notre manière d'être.

3 Si Bollnow ne prétend nullement élaborer une théorie architecturale inédite puisque la portée de son ouvrage est bien plus vaste, ce dernier fourmille cependant d'analyses susceptibles d'y contribuer, ce qui n'a échappé ni à Christian Norberg-Schulz (1971) dans Existence, Space and Architecture ni à Nold Egenter (1992) dans The Present Relevance of the Primitive in Architecture. Le premier propose une typologie des styles architecturaux au regard de l'espace vécu, tandis que le second élabore à sa suite une véritable anthropologie architecturale. Les développements de Bollnow qui intéresseront le plus les théoriciens et les praticiens de l'architecture se trouvent dans les pages consacrées à la maison dans le troisième chapitre. En effet, son exploration de l'espace vécu l'amène à distinguer une tension fondamentale et dialectique entre l'espace où l'on séjourne et celui où l'on voyage, entre d'un côté le mouvement du départ qui décentre et arrache, et de l'autre celui du retour qui rassemble et fixe dans 
un lieu d'attache, ce qu'il appelle le double mouvement de la vie, dont le réveil et l'endormissement sont les deux pulsations biologiques. Or, si les développements sur l'espace hodologique, c'est-à-dire l'expérience du voyage développée par Kurt Lewin (1934), en présentent une compréhension que l'on peut trouver réductrice, dans la mesure où ils soulignent une homogénéisation du paysage par les réseaux de mobilité et une désindividualisation du voyageur, au mépris de tous les grands textes littéraires sur la question, que ce soit le Voyage en Orient de Gustave Flaubert (2006) ou Sur la route de Jack Kerouac (1969), les analyses sur l'espace intime s'avèrent, pour leur part, tout à fait saisissantes. Une grande attention est portée aux différentes ouvertures et aux espaces de transition qui doivent empêcher l'espace protecteur de devenir une prison, sans nier pour autant sa fonction de point d'ancrage dans le monde. A ce titre, la critique, au nom de ce que habiter veut dire, des grandes baies vitrées, si prisées par le Mouvement Moderne, ainsi que de «la machine à habiter » en général, éclairera non seulement les historiens et les praticiens de l'architecture mais de manière plus large tout usager qui comprend que l'habitation déborde la simple fonction de l'abri et traduit de manière plus fondamentale le rapport privilégié de l'homme à l'espace. La réflexion sur la Wohnlichkteit (homeliness en anglais), terme sans équivalent en français, offre une magistrale leçon de la manière dont la réflexion sur les ambiances peut offrir une perspective précise sur l'environnement physique et ses modes de conception: qu'est-ce qui rend une maison wohnlich, ce que l'on peut traduire, faute de mieux, par commode à habiter?

4 Mais si les analyses phénoménologiques de Bollnow sur l'aménagement de la maison et la disposition des pièces, à partir de la mise en évidence du caractère structuré de l'espace autour d'un centre particulier, se révèlent aussi fines que profondes, le lecteur philosophe pourra cependant regretter la faiblesse de son explication anthropologique. En effet, Bollnow reprend les analyses développées par Gaston Bachelard dans sa Poétique de l'espace pour mettre l'accent sur la fonction protectrice de la maison, espace de fixation du bonheur dans lequel les forces adverses sont tenues à l'écart. Or une attention plus poussée à l'analytique du Dasein, à laquelle il se réfère pourtant un certain nombre de fois, lui aurait permis de justifier ce besoin de sécurité à partir d'un des existentiaux mis au jour par Heidegger : la facticité (Heidegger, 1986, p. 191). C'est parce que l'homme est jeté, de manière contingente, dans un monde qu'il n'a pas choisi qu'il va chercher à résorber ce sentiment d'étrangeté en y trouvant une place. Les critiques que Bollnow adresse un certain nombre de fois à l'existentialisme montrent qu'il méconnaît l'articulation essentielle entre l'être-jeté et l'être-au-monde, en considérant qu'elles sont au contraire contradictoires. Ainsi les descriptions de l'espace quotidien qui forment le cœur de l'ouvrage, si riches, aiguës et profondes soient-elles, gagnent-elles à être éclairées par certaines analyses de Etre et Temps, ainsi que de «Bâtir, habiter, penser ». Loin de demeurer un éternel étranger au monde, l'homme cherche au contraire à s'aménager un espace et en ce sens, la philosophie existentialiste vient renforcer les développements de Bollnow.

5 Le lecteur attentif au monde sensible et à la manière dont son prisme permet de renouveler l'appréhension des concepts traditionnels appréciera particulièrement la distinction entre différents types d'espaces, opérée dans le quatrième chapitre afin de récuser une compréhension homogène, réductrice et qualitativement neutre de l'environnement: notamment la tension entre l'espace diurne et l'espace nocturne, présentés comme des espaces de sensations différentes, vision d'un côté, ouïe et toucher de l'autre; ou encore l'espace thymique et ses modulations, pour reprendre la 
traduction française consacrée de l'expression de gestimmter Raum de Ludwig Binswanger, rendue dans le texte anglais par mood space. Le lien entre la manière dont l'espace est perçu et l'état émotif et psychologique de l'observateur montre qu'il faut dépasser le dualisme entre objectif et subjectif pour faire de l'humeur un concept clé dans la compréhension de l'espace expérimenté. Bollnow nous invite, de la sorte, à envisager l'humeur propre à chaque espace, susceptible de contaminer la personne présente en son sein, et l'humeur de l'homme déteignant, à son tour, sur son environnement, comme deux aspects d'un même phénomène, ce qu'il développe par la suite sous les termes de " conditions atmosphériques de l'expérience de l'espace ». Si le terme d'ambiance n'apparaît pas sous sa plume, nul doute que cette dernière expression, étayée par des références à Goethe, Binswanger, Tellenbach et Nietzsche, n'en offre un éclairage saisissant.

On l'aura compris, la traduction anglaise de Mensch und Raum met à la disposition d'un large public un texte charnière de l'anthropologie de l'espace. S'appuyant sur la compréhension phénoménologique de l'espace, il déborde le champ de la philosophie pour mettre ses concepts au service d'une analyse aussi profonde que minutieuse des différentes composantes de l'espace vécu et des manières dont nous l'expérimentons. En ce sens, la complexité de la réflexion théorique ne verse jamais dans l'abstraction et se révèle toujours portée par «le sol raboteux » de l'ordinaire : elle s'élabore pas à pas dans le soin mis à faire varier les perspectives et les points de vue. Intéressant la psychologie environnementale, l'architecture et le design urbain, Mensch und Raum montre que l'approche sensible de l'espace n'est pas seulement une possibilité mais une véritable nécessité.

\section{BIBLIOGRAPHIE}

Aristote. 2000. Physique. Paris : GF Flammarion.

Bachelard, Gaston. 1957. Poétique de l'espace. Paris : PUF.

Bergson, Henri. 2009 [1934]. La pensée et le mouvant. Paris : PUF.

Bergson, Henri. 2007 [1889]. Essai sur les données immédiates de la conscience. Paris : PUF.

Bollnow, Otto Friedrich. 2011 [1963]. Human Space. London: Hyphen Press.

Dürckheim, Karlfried Graf. 1932. Untersuchungen zum gelebten Raum. Neue Psychologische

Studien. vol. 6, n 4, p. 383-480.

Egenter, Nold. 1992. The Present Relevance of the Primitive in Architecture. London: Structura Mundi.

Flaubert, Gustave. 2006 [1849-1851]. Voyage en Orient. Paris : Gallimard.

Grimm, Jacob and Wilhelm. 1854. Deutsches Wörterbuch. Leipzig: Hirzel.

Heidegger, Martin. 1986 [1927]. Etre et temps. Paris : Authentica.

Kerouac, Jack. 1969 [1957]. Sur la Route. Paris : Gallimard. 
Lewin, Kurt. 1934. Der Richtungsbegriff in der Psychologie. Der spezielle und allgemeine hodologische Raum. Psychologische Forschung, vol. 19, p. 249-299.

Norberg-Schulz, Christian. 1971. Existence, Space and Architecture. London: Praeger.

\section{AUTEURS}

\section{CÉLINE BONICCO-DONATO}

Céline Bonicco-Donato est maître-assistante en sciences humaines et sociales à l'Ecole Nationale Supérieure d'Architecture de Grenoble et chercheuse au CRESSON (UMR Ambiances Architecturales et Urbaines). Agrégée et docteure en philosophie, elle travaille à l'interface de cette discipline et des sciences sociales sur la question de l'espace public en s'appuyant d'une part sur l'interactionnisme réaliste de Erving Goffman et d'autre part sur la philosophie des sentiments moraux de David Hume.

celine.bonicco@grenoble.archi.fr 